\title{
The B7 family molecules in oral squamous cell carcinoma: a systematic review. Part I: B7-H1 (PD-L1) and B7-DC (PD-L2)
}

\author{
Anna Starzyńska ${ }^{1}$, Aleksandra Sejda², tukasz Adamski ${ }^{1}$, Paulina Adamska1 ${ }^{1}$ Rafał Pęksa ${ }^{3}$, \\ Monika Sakowicz-Burkiewicz ${ }^{4}$, Piotr Wychowański ${ }^{5}$, Barbara A. Jereczek-Fossa ${ }^{6,7}$
}

'Department of Oral Surgery, Medical University of Gdansk, Gdansk, Poland 2Department of Pathomorphology, University of Warmia and Mazury, Olsztyn, Poland ${ }^{3}$ Department of Pathomorphology, Medical University of Gdansk, Gdansk, Poland ${ }^{4}$ Department of Molecular Medicine, Medical University of Gdansk, Gdansk, Poland ${ }^{5}$ Department of Oral Surgery, Medical University of Warsaw, Warsaw, Poland ${ }^{6}$ Division of Radiotherapy, IEO European Institute of Oncology, IRCCS, Milan, Italy ${ }^{7}$ Department of Oncology and Hemato-oncology, University of Milan, Milan, Italy

\begin{abstract}
Introduction: Oral squamous cell carcinoma (OSCC) is the most common cancerous lesion in the oral cavity. During recent years, no significant reduction in the survival rate has been observed.

Aim: To systematically review the literature and to summarise correlations between B7 family proteins and prognosis in OSCC.

Material and methods: A systematic review of the literature about B7-H1 (PD-L1) and B7-DC (PD-L2) was carried out, following the Preferred Reporting Items for Systematic Reviews and Meta-Analyses (PRISMA) guidelines. Thirty-six articles published before 22 May 2020 were included in the systematic review.

Results: The biggest study group consisted of 305 patients and the smallest -10 patients. PD-L1 proved to be a prognostic factor in patients with OSCC. Immunohistochemistry was the most commonly used diagnostic method. Conclusions: Any mutations in the gene encoding PD-L1 and quantitative or functional changes in the status of PD-L1 may be important in the prognosis of OSCC.
\end{abstract}

Key words: squamous cell carcinoma, oral cavity, B7 antigens.

\section{Introduction}

Ninety-five percent of oral neoplasms are diagnosed as squamous cell carcinoma (oral squamous cell carcinoma-OSCC). In 2017, about 390,000 new cases around the world were diagnosed (lip and oral cavity). Men suffer more often than women, especially in the sixth decade of life [1-4]. This type of cancer mainly affects people living in South-Central Asia, Central and Eastern Europe and in Malaysia [1, 2]. Smoking, betel chewing, alcohol abuse and HPV infection are the main risk factors. There is a systematic increase in the prevalence among women and patients under 50 years of age [3, 4].

In a properly functioning organism, damaged cells undergo apoptosis, a process in which various elements of the immune system participate. The main role is played by T and NK (natural killers) lymphocytes. In tumour pathology, cells gain unlimited ability to divide and survive as a result of changes in their genetic material. Lymphocyte function is the result of a balance between up-regulation (stimulation) and down-regulation (inhibition). Some types of cancers exhibit immunogenicity, i.e. the ability to induce an anti-cancer reaction. There are several molecular mechanisms involved in the regulation of the microenvironment of cancer cells and in the protection against the attack of the immune system [5]. Activation of T cells requires two signals. The first one is T cell receptor (TCR) activation - the major histocompatibility complex (MHC) on antigen-presenting cells (APC).

Address for correspondence: Anna Starzyńska, Department of Oral Surgery, Medical University of Gdansk, 7 Dębinki St, 80-211 Gdansk, Poland, phone: +48 5834915 71, e-mail: ast@gumed.edu.pl Received: 2.07.2020, accepted: 3.08.2020. 
The second signal is based on co-stimulatory molecules such as the B7-CD28 pathway [6].

The B7/CD28 family receptors are found on cells of the immune system. These are tumour infiltrating lymphocytes (TILs). Ligands (B7 proteins) are expressed on APC cells, immune cells, epithelial cells, osteoblasts, fibroblasts and others. In addition, the presence of ligands on tumour cells was detected [7]. The B7 family includes proteins and ligands as shown in Figure 1. The B7/CD28 pathway influences the regulation of the immune response by limiting both time and strength of the inflammatory response. Although the co-stimulation mechanism of the B7/CD28 pathway is not known, monoclonal antibodies are currently used in targeted therapies of malignant tumours, autoimmune and infectious diseases [7-9].

\section{Aim}

The aim of this paper was to collect and review the B7 family proteins as prognostic factors in OSCC and to describe their role in aggressive disease progression. This particular study includes B7-H1 (PD-L1) and B7-DC (PD-L2) proteins.

\section{Material and methods}

For this review, a systematic search of the literature was conducted in the PubMed, Web of Science, Scopus, Embase, Cochrane Library, and Google Scholar databases to identify papers containing data about the B7 family proteins in OSCC. The PRISMA guidelines (Preferred Reporting Items for Systematic Reviews and Meta-Analyses) were used [10].

PICO for study characteristics was used ( $\mathrm{P}$ - patient/ population/problem; I - intervention/exposure; C - comparison; O - outcome):

P: at least 10 patients with primary oral squamous cell carcinoma;

I: protein expression evaluation;

C: not required;

$\mathrm{O}$ : B7 family proteins as prognostic factors in OSCC - only B7-H1 (PD-L1) and B7-DC (PD-L2) proteins.
Inclusion criteria for papers were as follows: at least 10 patients with primary oral squamous cell carcinoma, B7 protein expression evaluation, study conducted only on humans, in the English language, prospective and retrospective studies, clinical studies and immunohistochemical evaluation of B7 protein. Articles were excluded if specimens derived from OSCC recurrences (not primary tumours), was conducted on cell lines, was not conducted on humans, the study group consisted of less than 10 patients, non-B7 family protein expression was evaluated or the study was not in English. Duplicate records as well as letters and papers that did not contain significant information were also excluded.

A retrospective analysis of articles on the $B 7$ family proteins as risk factors in OSCC published from 2011 to 22 May 2020, was performed. Key words: "B7 family and oral cancer/OSCC/oral squamous cell carcinoma", "PD-1/ PD-L1/PD-L2 pathway and oral cancer/OSCC/oral squamous cell carcinoma”, "B7-H1/PD-L1/CD274/PDCD1LG1/ B7H1/B7-H/PDCD1L1/PDCD1LG1/PDL1 and oral cancer/ OSCC/oral squamous cell carcinoma", "B7-DC/CD273/ PDCD1LG2/B7DC/Btdc/PDCD1L2/PDL2/bA574F11.2 and oral cancer/OSCC/oral squamous cell carcinoma", "PD1 signal transduction and oral cancer/OSCC/oral squamous cell carcinoma" were used. Articles were screened and sorted based on titles and abstracts. Then articles were evaluated for eligibility. Data extracted from those records were analysed in detail. The following pieces of information were collected: total patient number, occurrence of B7 family alterations in OSCC, correlations with age, gender, grading, primary tumour size (T stage), nodal metastases ( $\mathrm{N}$ stage), staging, prognostic significance and diagnostic methods (immunohistochemistry and other methods). For randomized studies, the Cochrane Collaboration tool [11] and the methodological index for non-randomized studies (MINORS) were used [12]. The ideal global score for non-comparative studies is 16 and for comparative is 24 [12].

\section{Statistical analysis}

Statistical analysis was carried out using the Statistica 13.3 (StatSoft Inc. Tulsa, United States), licensed by the Gdansk Medical University.

\begin{tabular}{|c|}
\hline B7/CD28 family \\
\hline \hline Ligands \\
\hline
\end{tabular}
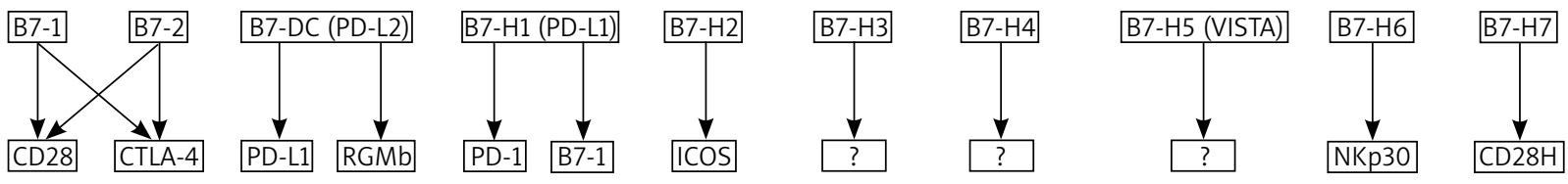

Figure 1. The B7 family ligands and receptors (? - unknown receptor) 


\section{Results}

In the first step of selection 923 references were identified. 533 records were selected after exclusion of the duplicates. Eventually, 36 articles were included in the systematic review (Figure 2) [13-48]. Three studies involved PD-L2 while 35 articles were on PD-L1. The first identified study was published in 2011. Table 1 shows the articles included in the analysis [13-48].

All presented papers were observational and cohortbased studies (Tables 2, 3). There were no randomized controlled trials. $86.11 \%$ were retrospective $(n=31)$, $11.11 \%$ of the studies were prospective $(n=4)$ and $2.78 \%$ were retro-prospective $(n=1)$.

The biggest study group consisted of 305 patients and the smallest - 10 patients. In total, 3170 patients (excluding duplicates) were analysed in the studies. The occurrence of protein expression was as follows: PD-L1 18.4-100\% and PD-L2 - 23.8-100\%. PD-L1 protein was associated with gender $[16,18,35,41,44]$, grading $[19,48]$, primary tumour size (T stage) [21, 29, 30] and metastases in lymph nodes [17, 25, 39, 48]. Staging was correlated

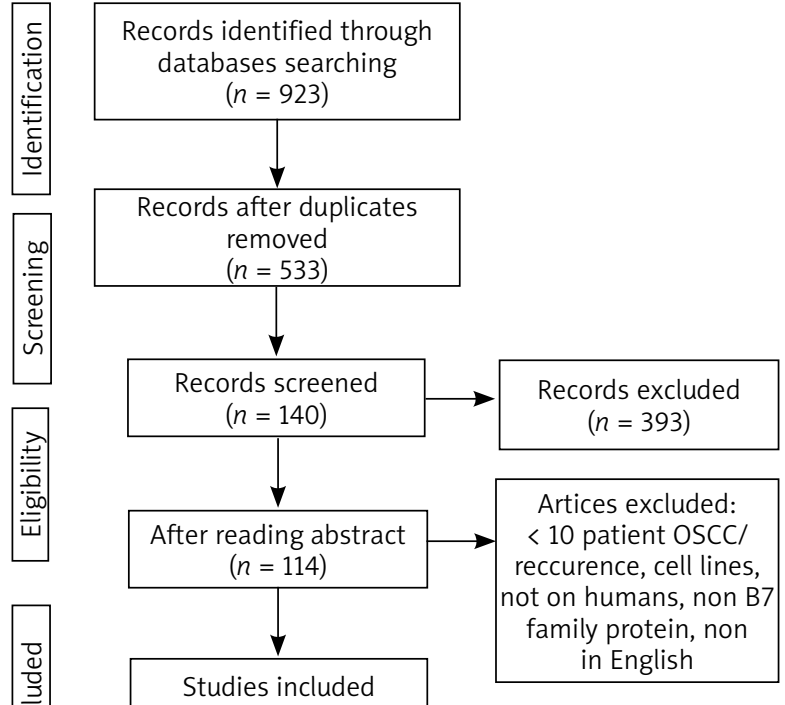
in quantitative synthesis $(n=36)$

Figure 2. PRISMA flow diagram of study selection

Table 1. B7 family genetic alterations in oral squamous cell carcinomas: a review of current studies

\begin{tabular}{|c|c|c|c|c|c|c|c|c|c|c|c|c|}
\hline \multirow[t]{2}{*}{ No. } & \multirow[t]{2}{*}{ Reference } & \multirow{2}{*}{$\begin{array}{l}\text { Study } \\
\text { type }\end{array}$} & \multirow{2}{*}{$\begin{array}{c}\text { Total } \\
\text { patient } \\
\text { number }\end{array}$} & \multirow{2}{*}{$\begin{array}{c}\text { Occurrence } \\
\text { of protein } \\
\text { expression } \\
n \text { patients (\%) }\end{array}$} & \multicolumn{7}{|c|}{ Correlations } & \multirow{2}{*}{ 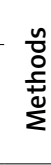 } \\
\hline & & & & & Age & Gender & Grading & $\begin{array}{l}\text { Primary } \\
\text { tumour size } \\
\text { (T stage) }\end{array}$ & $\begin{array}{c}\text { Nodal } \\
\text { metastases } \\
\text { (N stage) }\end{array}$ & Staging & $\begin{array}{l}\text { Prognostic } \\
\text { significance }\end{array}$ & \\
\hline \multicolumn{13}{|c|}{ B7-H1 (PD-L1): } \\
\hline 1 & $\begin{array}{l}\text { Cui et al., } \\
2020 \text { [13] }\end{array}$ & $\mathrm{O}, \mathrm{R}, \mathrm{C}$ & 34 & No data & $\mathrm{NE}$ & NE & $\mathrm{NE}$ & $\mathrm{NE}$ & NE & $\mathrm{NE}$ & NE & $\mathrm{IHC}$ \\
\hline 2 & $\begin{array}{c}\text { Meehan } \\
\text { et al., } 2020 \text { [14] }\end{array}$ & O, R, C & 67 & No data & $\mathrm{NE}$ & NE & NE & $\mathrm{NE}$ & $\mathrm{NE}$ & NE & NS & $\mathrm{IHC}$ \\
\hline 3 & $\begin{array}{l}\text { Quan et al., } \\
2020 \text { [15] }\end{array}$ & O, R, C & 159 & No data & $\mathrm{NE}$ & $\mathrm{NE}$ & $\mathrm{NE}$ & $\mathrm{NE}$ & $\mathrm{NE}$ & $\mathrm{NE}$ & $\begin{array}{c}\text { OS - NS } \\
p=0.742\end{array}$ & $\mathrm{IHC}$ \\
\hline 4 & $\begin{array}{c}\text { Wilms et al., } \\
2020 \text { [16] }\end{array}$ & $\begin{array}{c}\text { O, R-P, } \\
\text { C }\end{array}$ & 101 & 80 (79.2\%) & $\begin{array}{c}\text { NS } \\
p=0.494\end{array}$ & $p=0.019$ & NE & $\begin{array}{c}\text { NS } \\
p=0.929\end{array}$ & $\begin{array}{c}\text { NS } \\
p=0.286\end{array}$ & $\begin{array}{c}\text { NS } \\
p=0.888\end{array}$ & $\begin{array}{c}\text { OS }-S \\
p=0.021 \\
\text { DFS }-S \\
p=0.020\end{array}$ & $\mathrm{IHC}$ \\
\hline 5 & $\begin{array}{c}\text { Zhao et al., } \\
2020 \text { [17] }\end{array}$ & $\mathrm{O}, \mathrm{R}, \mathrm{C}$ & 46 & $30(65.2 \%)$ & $\begin{array}{c}\text { NS } \\
p=0.829\end{array}$ & $\begin{array}{c}\text { NS } \\
p=0.956\end{array}$ & $\begin{array}{c}\text { NS } \\
p=0.806\end{array}$ & $\begin{array}{c}\text { NS } \\
p=0.052\end{array}$ & $p=0.009$ & $p=0.011$ & NE & $\mathrm{IHC}$ \\
\hline 6 & $\begin{array}{l}\text { Ahmadi } \\
\text { et al., } \\
2019[18]\end{array}$ & $\mathrm{O}, \mathrm{R}, \mathrm{C}$ & 255 & 70 (27.5\%) & $\begin{array}{c}\text { NS } \\
p=0.610\end{array}$ & $p=0.005$ & $\begin{array}{c}\text { NS } \\
p=0.760\end{array}$ & $\begin{array}{c}\text { NS } \\
p=0.260\end{array}$ & $\begin{array}{c}\text { NS } \\
p=0.660\end{array}$ & $\mathrm{NE}$ & $\begin{array}{c}\text { OS }-\mathrm{NS} \\
p=0.482 \\
\mathrm{DSS}-\mathrm{NS} \\
p=0.864 \\
\mathrm{DFS}-\mathrm{NS} \\
p=0.731\end{array}$ & $\mathrm{IHC}$ \\
\hline 7 & $\begin{array}{c}\text { Chen et al., } \\
2019 \text { [19] }\end{array}$ & $\mathrm{O}, \mathrm{P}, \mathrm{C}$ & 41 & $40(97.6 \%)$ & $\begin{array}{c}\text { NS } \\
p=0.088\end{array}$ & $\begin{array}{c}\text { NS } \\
p=0.857\end{array}$ & $p=0.010$ & $\begin{aligned} \mathrm{NS} \\
p=0.9414\end{aligned}$ & $\begin{array}{c}\text { NS } \\
p=0.147\end{array}$ & NE & $\begin{array}{c}\text { OS }-\mathrm{NS} \\
p=0.696 \\
\mathrm{DFS}-\mathrm{NS} \\
p=0.210\end{array}$ & $\mathrm{IHC}$ \\
\hline 8 & $\begin{array}{c}\text { de Vincente } \\
\text { et al., } 2019 \text { [20] }\end{array}$ & $\mathrm{O}, \mathrm{R}, \mathrm{C}$ & 125 & $4(3.2 \%)$ & NE & $\begin{array}{c}\text { NS } \\
p=0.530\end{array}$ & $\begin{array}{c}\text { NS } \\
p=1.000\end{array}$ & $\begin{array}{c}\text { NS } \\
p=0.580\end{array}$ & $\begin{array}{c}\text { NS } \\
p=0.570\end{array}$ & $\begin{array}{c}\text { NS } \\
p=0.350\end{array}$ & $\begin{array}{c}\text { DFS }-S \\
p=0.030\end{array}$ & $\mathrm{IHC}$ \\
\hline 9 & $\begin{array}{c}\text { Kouketsu } \\
\text { et al., } 2019 \text { [21] }\end{array}$ & $\mathrm{O}, \mathrm{R}, \mathrm{C}$ & 106 & 73 (68.9\%) & $\begin{array}{c}\text { NS } \\
p=0.999\end{array}$ & $\begin{array}{c}\text { NS } \\
p=511\end{array}$ & $\mathrm{NE}$ & $p=0.018$ & $\begin{array}{c}\text { NS } \\
p=0.472\end{array}$ & $p=0.010$ & OS - NS & $\mathrm{IHC}$ \\
\hline
\end{tabular}


Table 1. Cont.

\begin{tabular}{|c|c|c|c|c|c|c|c|c|c|c|c|c|}
\hline \multirow[t]{2}{*}{ No. } & \multirow[t]{2}{*}{ - Reference } & \multirow{2}{*}{$\begin{array}{l}\text { Study } \\
\text { type }\end{array}$} & \multirow{2}{*}{$\begin{array}{c}\text { Total } \\
\text { patient } \\
\text { number }\end{array}$} & \multirow{2}{*}{$\begin{array}{c}\text { Occurrence } \\
\text { of protein } \\
\text { expression } \\
n \text { patients (\%) }\end{array}$} & \multicolumn{7}{|c|}{ Correlations } & \multirow[b]{2}{*}{$\begin{aligned} \frac{n}{0} \\
\frac{0}{ \pm} \\
\sum\end{aligned}$} \\
\hline & & & & & Age & Gender & Grading & $\begin{array}{l}\text { Primary } \\
\text { tumour size } \\
\text { (T stage) }\end{array}$ & $\begin{array}{c}\text { Nodal } \\
\text { metastases } \\
\text { (N stage) }\end{array}$ & Staging & $\begin{array}{l}\text { Prognostic } \\
\text { significance }\end{array}$ & \\
\hline 10 & $\begin{array}{c}\text { Takahashi } \\
\text { et al., } 2019 \text { [22] }\end{array}$ & O, R, C & 77 & $46(60 \%)$ & $\begin{array}{c}\text { NS } \\
p=0.880\end{array}$ & $\begin{array}{c}\text { NS } \\
p=0.360\end{array}$ & $\begin{array}{c}\text { NS } \\
p=0.420\end{array}$ & $\begin{array}{c}\text { NS } \\
p=0.750\end{array}$ & $\begin{array}{c}\text { NS } \\
p=0.790\end{array}$ & $\begin{array}{c}\text { NS } \\
p=0.580\end{array}$ & $\begin{array}{c}\text { PFS - NS } \\
p=0.540 \\
\text { OS - NS } \\
p=0.920\end{array}$ & $\mathrm{IHC}$ \\
\hline 11 & $\begin{array}{c}\text { Tojyo } \\
\text { et al., } 2019 \text { [23] }\end{array}$ & ] $\mathrm{R}, \mathrm{C}$ & 48 & 44 (91.7\%) & $\begin{array}{c}\text { NS } \\
p=1.000\end{array}$ & $\begin{array}{c}\text { NS } \\
p=1.000\end{array}$ & $\begin{array}{c}\text { NS } \\
p=0.540\end{array}$ & $\begin{array}{c}\text { NS } \\
p=0.520\end{array}$ & $\begin{array}{c}\text { NS } \\
p=1.000\end{array}$ & NE & $\begin{array}{l}\text { DFS - NS } \\
p=0.185\end{array}$ & $\mathrm{IHC}$ \\
\hline 12 & $\begin{array}{c}\text { Hanna } \\
\text { et al., } 2018 \text { [24] }\end{array}$ & $\mathrm{O}, \mathrm{R}, \mathrm{C}$ & $\begin{array}{c}81 \text { (32 } \\
\text { females) }\end{array}$ & $\begin{array}{l}28 \text { females } \\
(87 \%)\end{array}$ & $\mathrm{NE}$ & $\mathrm{NE}$ & $\mathrm{NE}$ & $\mathrm{NE}$ & $\mathrm{NE}$ & $\mathrm{NE}$ & $\begin{array}{c}\text { Female OS } \\
-S \\
p<0.001\end{array}$ & $\mathrm{IHC}$ \\
\hline 13 & $\begin{array}{c}\text { Maruse } \\
\text { et al., } 2018 \text { [25] }\end{array}$ & O, R, C & 97 & 63 (64.9\%) & $\begin{array}{c}\text { NS } \\
p>0.050\end{array}$ & $\begin{array}{c}\text { NS } \\
p>0.050\end{array}$ & $\begin{array}{c}\text { NS } \\
p>0.050\end{array}$ & $\begin{array}{c}\text { NS } \\
p>0.050\end{array}$ & $\begin{array}{c}\mathrm{S} \\
p=0.050\end{array}$ & $\begin{array}{c}\text { NS } \\
p>0.050\end{array}$ & $\begin{array}{c}\text { NS } \\
p>0.050\end{array}$ & $\mathrm{IHC}$ \\
\hline 14 & $\begin{array}{l}\text { Stasikowska- } \\
\text { Kanicka } \\
\text { et al., } 2018 \text { [26] }\end{array}$ & O, R, C & 78 & 62 (79\%) & NE & NE & NE & NE & NE & NE & $\begin{array}{c}\mathrm{PP}-\mathrm{S} \\
p<0.011\end{array}$ & $\mathrm{IHC}$ \\
\hline 15 & $\begin{array}{l}\text { Stasikowska- } \\
\text { Kanicka } \\
\text { et al., } 2018 \text { [27] }\end{array}$ & O, R, C & 70 & 67 (96\%) & $\mathrm{NE}$ & $\mathrm{NE}$ & $\mathrm{NE}$ & NE & $\mathrm{NE}$ & NE & NE & $\mathrm{IHC}$ \\
\hline 16 & $\begin{array}{l}\text { Udeabor } \\
\text { et al., } 2018 \text { [28] }\end{array}$ & ] $\mathrm{O}, \mathrm{R}, \mathrm{C}$ & 20 & No data & NE & NE & NE & NE & NE & NE & NE & $\mathrm{IHC}$ \\
\hline 17 & $\begin{array}{l}\text { Wirsing } \\
\text { et al., 2018 [29] }\end{array}$ & ] $\mathrm{O}, \mathrm{R}, \mathrm{C}$ & 75 & 45 (60\%) & NE & NE & NE & $\begin{array}{c}\mathrm{S} \\
p=0.024\end{array}$ & NS & NE & $\begin{array}{l}\text { DSD - NS } \\
p=0.207\end{array}$ & $\mathrm{IHC}$ \\
\hline 18 & $\begin{array}{l}\text { Ahn et al., } \\
2017 \text { [30] }\end{array}$ & $\mathrm{O}, \mathrm{R}, \mathrm{C}$ & 68 & 45 (66\%) & $\begin{array}{c}\text { NS } \\
p>0.050\end{array}$ & $\begin{array}{c}\text { NS } \\
p>0.050\end{array}$ & NE & $\begin{array}{c}\mathrm{S} \\
p=0.002\end{array}$ & $\begin{array}{c}\text { NS } \\
p=0.648\end{array}$ & $\begin{array}{c}\mathrm{S} \\
p=0.010\end{array}$ & $\begin{array}{c}\text { DFS }- \text { NS } \\
p=0.070 \\
\text { OS }-S \\
p=0.039\end{array}$ & $\mathrm{IHC}$ \\
\hline 19 & $\begin{array}{l}\text { Feng et al., } \\
2017 \text { [31] }\end{array}$ & $\mathrm{O}, \mathrm{R}, \mathrm{C}$ & 119 & No data & NE & NE & NE & NE & NE & NE & $\begin{array}{c}\text { OS }-S \\
p=0.007\end{array}$ & $\mathrm{IHC}$ \\
\hline 20 & $\begin{array}{l}\text { Foy et al., } \\
2017 \text { [32] }\end{array}$ & $\mathrm{O}, \mathrm{R}, \mathrm{C}$ & 44 & No data & $\mathrm{NE}$ & NE & $\mathrm{NE}$ & $\mathrm{NE}$ & $\mathrm{NE}$ & NE & NE & $\mathrm{IHC}$ \\
\hline 21 & $\begin{array}{c}\text { Groeger } \\
\text { et al., } 2017 \text { [33] }\end{array}$ & O, P, C & 15 & 15 (100\%) & NE & NE & NE & NE & NE & NE & NE & $\mathrm{IHC}$ \\
\hline 22 & $\begin{array}{l}\text { Hirai et al., } \\
2017 \text { [34] }\end{array}$ & $\mathrm{O}, \mathrm{P}, \mathrm{C}$ & 24 & No data & NE & NE & $\begin{array}{c}\text { NS } \\
0.145\end{array}$ & $\begin{array}{c}\text { NS } \\
p=0.873\end{array}$ & $\begin{array}{c}\text { NS } \\
p=0.542\end{array}$ & NE & NE & $\mathrm{IHC}$ \\
\hline 23 & $\begin{array}{c}\text { Kogashiwa } \\
\text { et al., } 2017 \text { [35] }\end{array}$ & O, $\mathrm{R}, \mathrm{C}$ & 84 & 44 (52\%) & $\begin{array}{c}\text { NS } \\
p=0.492\end{array}$ & $\begin{array}{c}\mathrm{S} \\
p=0.010\end{array}$ & NE & $\begin{array}{c}\text { NS } \\
p=0.613\end{array}$ & $\begin{array}{c}\text { NS } \\
p=0.734\end{array}$ & $\begin{array}{c}\text { NS } \\
p=0.235\end{array}$ & $\begin{array}{c}\text { OS }-S \\
p=0.006 \\
\text { PFS }-S \\
p=0.024\end{array}$ & $\mathrm{IHC}$ \\
\hline 24 & $\begin{array}{c}\text { Kubota et al., } \\
2017 \text { [36] }\end{array}$ & O, R, C & 46 & No data & $\mathrm{NE}$ & NE & $\mathrm{NE}$ & $\mathrm{NE}$ & NE & NE & NE & $\mathrm{IHC}$ \\
\hline 25 & $\begin{array}{l}\text { Mattox et al., } \\
2017 \text { [37] }\end{array}$ & O, R, C & 53 & 39 (73\%) & $\mathrm{NE}$ & $\mathrm{NE}$ & $\mathrm{NE}$ & $\mathrm{NE}$ & $\mathrm{NE}$ & NE & $\begin{array}{c}\text { OS - NS } \\
p=0.830\end{array}$ & $\mathrm{IHC}$ \\
\hline 26 & $\begin{array}{c}\text { Takakura } \\
\text { et al., } 2017 \text { [38] }\end{array}$ & O, $\mathrm{R}, \mathrm{C}$ & $\begin{array}{c}10 \\
\text { (patients } \\
\text { without } \\
\text { chemothe- } \\
\text { rapy) }\end{array}$ & $8(80 \%)$ & NE & NE & NE & NE & NE & NE & NE & $\mathrm{IHC}$ \\
\hline 27 & $\begin{array}{l}\text { Troeltzsch } \\
\text { et al., } 2017 \text { [39] }\end{array}$ & O, R, C & 88 & 26 (29\%) & $\begin{array}{c}\text { NS } \\
p=0.349\end{array}$ & $\begin{array}{c}\text { NS } \\
p=0.579\end{array}$ & $\begin{array}{c}\text { NS } \\
p=0.157\end{array}$ & $\begin{array}{c}\text { NS } \\
p=0.831\end{array}$ & $\begin{aligned} & S \\
p= & 0.039\end{aligned}$ & NE & $\begin{array}{l}\text { DSS - NS } \\
p=0.937\end{array}$ & $\mathrm{IHC}$ \\
\hline
\end{tabular}


Table 1. Cont.

\begin{tabular}{|c|c|c|c|c|c|c|c|c|c|c|c|c|}
\hline \multirow[t]{2}{*}{ No. } & \multirow[t]{2}{*}{ Reference } & \multirow{2}{*}{$\begin{array}{l}\text { Study } \\
\text { type }\end{array}$} & \multirow{2}{*}{$\begin{array}{c}\text { Total } \\
\text { patient } \\
\text { number }\end{array}$} & \multirow{2}{*}{$\begin{array}{c}\text { Occurrence } \\
\text { of protein } \\
\text { expression } \\
n \text { patients (\%) }\end{array}$} & \multicolumn{7}{|c|}{ Correlations } & \multirow[b]{2}{*}{ 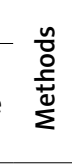 } \\
\hline & & & & & Age & Gender & Grading & $\begin{array}{c}\text { Primary } \\
\text { tumour size } \\
\text { (T stage) }\end{array}$ & $\begin{array}{c}\text { Nodal } \\
\text { metastases } \\
(\mathrm{N} \text { stage })\end{array}$ & Staging & $\begin{array}{l}\text { Prognostic } \\
\text { significance }\end{array}$ & \\
\hline 28 & $\begin{array}{l}\text { Weber et al., } \\
2017 \text { [40] }\end{array}$ & $\mathrm{O}, \mathrm{R}, \mathrm{C}$ & 45 & $\begin{array}{c}35 / 43 \\
(1.81 .4 \% \\
\text { increased } \\
\text { PD-L1_4); } \\
32 / 43 \\
(2.74 .4 \% \\
\text { increased } \\
\text { PD-L1_2) }\end{array}$ & NE & NE & $\begin{array}{c}\text { 1. G1 vs. } \\
\mathrm{G} 3-\mathrm{S} \\
p=0.020 \\
\text { 2. G1 vs. } \\
\mathrm{G} 3-\mathrm{S} \\
p=0.010\end{array}$ & $\begin{array}{c}\text { 1.NS } \\
p=0.370 \\
\text { 2. NS } \\
p=0.487\end{array}$ & $\begin{array}{c}1 . S \\
p<0.002 \\
2 . S \\
p=0.003\end{array}$ & $\begin{array}{c}\text { NS } \\
p>0.05\end{array}$ & NE & $\mathrm{IHC}$ \\
\hline 29 & $\begin{array}{l}\text { Satgunaseelan } \\
\text { et al., } 2016 \text { [41] }\end{array}$ & O, $\mathrm{R}, \mathrm{C}$ & 217 & 40 (18.4\%) & $\begin{array}{c}\text { NS } \\
p=0.493\end{array}$ & $\begin{array}{c}\mathrm{S} \\
p=0.013\end{array}$ & $\begin{array}{c}\text { NS } \\
p=0.060\end{array}$ & $\begin{array}{c}\text { NS } \\
p=0.550\end{array}$ & $\begin{array}{c}\text { NS } \\
p=0.900\end{array}$ & NE & $\begin{array}{c}\text { DSS - NS } \\
p=0.960 \\
\text { DFS - NS } \\
p=0.820 \\
\text { OS - NS } \\
p=0.930\end{array}$ & $\mathrm{IHC}$ \\
\hline 30 & $\begin{array}{l}\text { Straub et al., } \\
2016 \text { [42] }\end{array}$ & $\mathrm{O}, \mathrm{R}, \mathrm{C}$ & 80 & $36(45 \%)$ & $\begin{array}{c}\text { NS } \\
p>0.050\end{array}$ & $\begin{array}{c}\text { NS } \\
p>0.050\end{array}$ & $\begin{array}{c}\mathrm{NS} \\
p>0.050\end{array}$ & $\begin{array}{c}\text { NS } \\
p>0.050\end{array}$ & $\begin{array}{c}\text { NS } \\
p>0.050\end{array}$ & $\begin{array}{c}\text { NS } \\
p>0.050\end{array}$ & $\begin{array}{c}\text { OS }-\mathrm{S} \\
p=0.010 \\
\text { RFS } \\
p=0.050\end{array}$ & $\mathrm{IHC}$ \\
\hline 31 & $\begin{array}{l}\text { Chen et al., } \\
2015 \text { [43] }\end{array}$ & $\mathrm{O}, \mathrm{R}, \mathrm{C}$ & 218 & 139 (64\%) & NE & NE & NE & NE & NE & NE & $\begin{array}{c}\text { DFS - NS } \\
p=0.020 \\
\text { OS - NS } \\
p=0.110\end{array}$ & $\mathrm{IHC}$ \\
\hline 32 & $\begin{array}{l}\text { Lin et al., } \\
2015 \text { [44] }\end{array}$ & $\begin{array}{c}\mathrm{O}, \\
\mathrm{R}, \mathrm{C}\end{array}$ & 305 & $134(44 \%)$ & $\begin{array}{c}\text { NS } \\
p=0.124\end{array}$ & $\begin{array}{c}\mathrm{S} \\
p=0.006\end{array}$ & $\begin{array}{c}\text { NS } \\
p=0.326\end{array}$ & $\begin{array}{c}\text { NS } \\
p=0.316\end{array}$ & $\begin{array}{c}\text { NS } \\
p=0.736\end{array}$ & $\begin{array}{c}\text { NS } \\
p=0.804\end{array}$ & $\begin{array}{c}\text { OS - NS } \\
p=0.083\end{array}$ & $\mathrm{IHC}$ \\
\hline 33 & $\begin{array}{l}\text { Oliveira- } \\
\text { Costa et al., } \\
2015 \text { [45] }\end{array}$ & $\begin{array}{l}\mathrm{O}, \\
\mathrm{R}, \mathrm{C}\end{array}$ & 142 & 47/97 (49\%) & $\begin{array}{c}\text { NS } \\
p>0.050\end{array}$ & $\begin{array}{c}\text { NS } \\
p>0.050\end{array}$ & $\begin{array}{c}\text { NS } \\
p>0.050\end{array}$ & $\begin{array}{c}\text { NS } \\
p>0.050\end{array}$ & $\begin{array}{c}\text { NS } \\
p>0.050\end{array}$ & $\begin{array}{c}\text { NS } \\
p>0.050\end{array}$ & $\begin{array}{c}\text { DSS }-S \\
p=0.044\end{array}$ & $\mathrm{IHC}$ \\
\hline 34 & $\begin{array}{l}\text { Cho et al., } \\
2011 \text { [46] }\end{array}$ & $\mathrm{O}, \mathrm{R}, \mathrm{C}$ & 45 & 39 (87\%) & $\begin{array}{c}\text { NS } \\
p=0.787\end{array}$ & $\begin{array}{c}\text { NS } \\
p=0.745\end{array}$ & $\begin{array}{c}\text { NS } \\
p=0.158\end{array}$ & $\begin{array}{c}\text { NS } \\
p=0.393\end{array}$ & $\begin{array}{c}\text { NS } \\
p=0.433\end{array}$ & $\begin{array}{c}\text { NS } \\
p=0.736\end{array}$ & $\begin{array}{c}\text { OS - NS } \\
p=0.501\end{array}$ & $\mathrm{IHC}$ \\
\hline 35 & $\begin{array}{c}\text { Malaspina } \\
\text { et al., } 2011 \text { [47] }\end{array}$ & $O, R, C$ & 39 & No data & NE & $\mathrm{NE}$ & NE & NE & $\mathrm{NE}$ & NE & NE & $\mathrm{IHC}$ \\
\hline \multicolumn{13}{|c|}{ B7-DC (PD-L2): } \\
\hline 1 & $\begin{array}{l}\text { Weber et al., } \\
2019 \text { [48] }\end{array}$ & $\mathrm{O}, \mathrm{P}, \mathrm{C}$ & 48 & $28 / 36(77.8 \%)$ & NE & NE & $\begin{array}{c}\text { NS } \\
p=0.130\end{array}$ & $\begin{array}{c}\text { NS } \\
p=0.805\end{array}$ & $\begin{array}{c}\text { NS } \\
p=0.960\end{array}$ & NE & $\begin{array}{c}\text { NS } \\
p=0.400\end{array}$ & $\begin{array}{l}\text { RT- } \\
\text { qPCR }\end{array}$ \\
\hline 2 & $\begin{array}{c}\text { Groeger et al., } \\
2017 \text { [33] }\end{array}$ & $\mathrm{O}, \mathrm{P}, \mathrm{C}$ & 15 & 15 (100\%) & NE & $\mathrm{NE}$ & NE & NE & NE & NE & NE & $\mathrm{IHC}$ \\
\hline 3 & $\begin{array}{c}\text { Kogashiwa } \\
\text { et al., } 2017 \text { [35] }\end{array}$ & $\mathrm{O}, \mathrm{R}, \mathrm{C}$ & 84 & 20 (23.8\%) & $\begin{array}{c}\text { NS } \\
p=0.792\end{array}$ & $\begin{array}{c}\text { NS } \\
p=1.000\end{array}$ & NE & $\begin{array}{c}\text { NS } \\
p=0.373\end{array}$ & $\begin{array}{c}\text { NS } \\
p=0.449\end{array}$ & $\begin{array}{c}\mathrm{S} \\
p=0.011\end{array}$ & $\begin{array}{c}\text { PFS - NS } \\
p=0.350 \\
\text { OS - NS } \\
p=0.058\end{array}$ & $\mathrm{IHC}$ \\
\hline
\end{tabular}

$R$ - retrospective study, $P$ - prospective study, $R$ - $P$-retro-prospective study, $O$ - observational study, $C$ - cohort study, $S$ - significant, NE - not examined, NS - not significant, OS - overall survival, $P P$ - poor prognosis, DSS - disease-specific survival, DSD - disease-specific death, DFS - disease-free survival, PFS - progression-free survival, RFS - recurrence-free survival, IHC - immunohistochemistry, RT-qPCR - reverse transcription polymerase chain reaction.

with PD-L1 [17, 20, 30] and PD-L2 [33]. Only PD-L1 protein expression proved to be a prognostic factor. Overall survival [16, 24, 31, 35, 42], disease-free survival [16, 20, 45], progression-free survival [35], poor prognosis [26] and recurrence-free survival [42] were correlated with PD-L1 protein expression. Immunohistochemistry was the most commonly used diagnostic method.

\section{Discussion}

$\mathrm{B} 7$ protein family and the receptors PD-L1 (B7-H1)/PD-L2 (B7-DC)/PD-1

B7-H1/PD-L1 protein (programmed cell death 1 ligand 1/cluster of differentiation 274/CD274/PDCD1LG1/B7H1/ B7-H/PDCD1L1/PDCD1LG1/PDL1) is a type I membrane 
Anna Starzyńska, Aleksandra Sejda, Łukasz Adamski, Paulina Adamska, Rafał Pęksa, Monika Sakowicz-Burkiewicz, Piotr Wychowański, Barbara A. Jereczek-Fossa

Table 2. Results of MINORS for non-comparative studies

\begin{tabular}{|c|c|c|c|c|c|c|c|c|c|c|}
\hline & \multirow[t]{2}{*}{ Reference } & \multicolumn{9}{|c|}{ MINORS for non-comparative studies } \\
\hline & & 1 & 2 & 3 & 4 & 5 & 6 & 7 & 8 & Score \\
\hline 1 & Quan et al., 2020 [15] & 2 & 2 & 2 & 1 & 0 & 1 & 2 & 0 & 10 \\
\hline 2 & Ahmadi et al., 2019 [18] & 2 & 2 & 2 & 1 & 0 & 1 & 2 & 0 & 10 \\
\hline 3 & de Vincente et al., 2019 [20] & 1 & 2 & 2 & 0 & 0 & 0 & 2 & 0 & 7 \\
\hline 4 & Takahashi et al., 2019 [22] & 2 & 1 & 1 & 2 & 0 & 0 & 2 & 0 & 8 \\
\hline 5 & Tojyo et al., 2019 [23] & 2 & 1 & 2 & 2 & 0 & 1 & 2 & 0 & 10 \\
\hline 6 & Hanna et al., 2018 [24] & 2 & 1 & 2 & 2 & 0 & 1 & 2 & 0 & 10 \\
\hline 7 & Maruse et al., 2018 [25] & 2 & 1 & 2 & 2 & 0 & 1 & 2 & 0 & 10 \\
\hline 8 & Udeabor et al., 2018 [28] & 2 & 0 & 0 & 1 & 0 & 0 & 2 & 0 & 5 \\
\hline 9 & Wirsing et al., 2018 [29] & 2 & 2 & 1 & 2 & 0 & 0 & 2 & 1 & 10 \\
\hline 10 & Ahn et al., 2017 [30] & 2 & 1 & 2 & 2 & 0 & 2 & 2 & 0 & 11 \\
\hline 11 & Feng et al., 2017 [31] & 0 & 1 & 2 & 2 & 1 & 2 & 2 & 0 & 10 \\
\hline 12 & Foy et al., 2017 [32] & 2 & 2 & 2 & 2 & 0 & 1 & 2 & 0 & 11 \\
\hline 13 & Groeger et al., 2017 [33] & 2 & 1 & 2 & 1 & 0 & 0 & 2 & 0 & 8 \\
\hline 14 & Hirai et al., 2017 [34] & 2 & 1 & 0 & 0 & 0 & 0 & 2 & 0 & 5 \\
\hline 15 & Kogashiwa et al., 2017 [35] & 2 & 2 & 2 & 1 & 0 & 1 & 2 & 0 & 10 \\
\hline 16 & Kubota et al., 2017 [36] & 2 & 1 & 2 & 2 & 0 & 1 & 2 & 0 & 10 \\
\hline 17 & Mattox et al., 2017 [37] & 1 & 1 & 2 & 2 & 0 & 1 & 2 & 0 & 9 \\
\hline 18 & Takakura et al., 2017 [38] & 2 & 1 & 2 & 2 & 0 & 1 & 2 & 0 & 10 \\
\hline 19 & Troeltzsch et al., 2017 [39] & 2 & 2 & 2 & 0 & 0 & 1 & 2 & 0 & 9 \\
\hline 20 & Satgunaseelan et al., 2016 [41] & 2 & 2 & 2 & 2 & 0 & 1 & 2 & 0 & 11 \\
\hline 21 & Straub et al., 2016 [42] & 2 & 2 & 2 & 2 & 0 & 1 & 2 & 0 & 11 \\
\hline 22 & Chen et al., 2015 [43] & 2 & 2 & 2 & 2 & 0 & 1 & 2 & 0 & 11 \\
\hline 23 & Lin et al., 2015 [44] & 2 & 1 & 2 & 2 & 0 & 1 & 2 & 0 & 10 \\
\hline 24 & Oliveira-Costa et al., 2015 [45] & 2 & 2 & 2 & 2 & 0 & 2 & 2 & 0 & 12 \\
\hline 25 & Cho et al., 2011 [46] & 2 & 2 & 2 & 2 & 0 & 1 & 2 & 0 & 11 \\
\hline
\end{tabular}

1 - a clearly stated aim, 2 - inclusion of consecutive patients, 3 - prospective data collection, 4 - endpoints appropriate to the aim of the study, 5 - unbiased assessment of the study endpoint, 6 - follow-up period appropriate to the aim of the study, 7 -loss to follow up less than $5 \%, 8$ - prospective calculation of the study size; score: 0 -not reported, 1 -reported but inadequate, 2 -reported and adequate; the ideal global score for comparative studies is 16.

protein (mass $40 \mathrm{kDa}$ ) encoded by the CD274 gene on chromosome 9 (locus 9p24.1). The B7-H1 protein has genes parallel to B7-1 ligand in 21\%. PD-L1 has three domains: immunoglobulin constant-like domain (lgC; extracellular), the variable-like domain (IgV; extracellular) and homology domain for PD-1 [8, 49]. Intracellular structures are poorly studied. PD-L1 molecules are more prevalent than PD-L2. The PD-L1 protein suppresses the immune system [50-52]. PD-L1 protein is found on activated T lymphocytes, dendritic cells, B lymphocytes, NK cells, monocytes, macrophages, endothelial cells, epithelial cells, fibroblasts, mesenchymal stem cells, syncytiotrophoblasts, islets of Langerhans and neurons. The PD-L1 molecule plays a crucial role in the differentiation of regulatory $\mathrm{T}$ lymphocytes. The increase in its status is also associated with chronic inflammation and secretion of interferon $\gamma($ IFN- $\gamma)[8,33,43,53,54]$. It can affect the re- sults of treatment of hepatitis B and C [55]. The presence of the PD-L1 protein expression has been demonstrated in glioma, ovarian cancer, renal cancer, head and neck cancer, breast cancer, sigmoid cancer, pancreatic cancer, non-small cell lung cancer and melanoma $[53,56]$. Wang et al. demonstrated that PD-L1 positive expression was a prognostic factor for poor disease-specific survival in pancreatic carcinoma [53]. An increase in the response to anti-PD-1/PD-L1 therapies has been demonstrated in the treatment of lung cancer associated with smoking. An increased expression of PD-L1 refers to solid tumours, where it can serve as a defence of the tumour against the immune system [44]. In OSCC, PD-L1 protein expression was correlated with gender [16, 18, 35, 41, 44], grade $[19,48]$, stage $[17,21,30]$, tumour size $[21,29,30]$, nodal metastases [25, 26, 39-42], distant metastases [25, 26], localisation [41], vascular invasion [28], positive TILs infil- 
Table 3. Results of MINORS for comparative studies

\begin{tabular}{|c|c|c|c|c|c|c|c|c|c|c|c|c|c|c|}
\hline & \multirow[t]{2}{*}{ Reference } & \multicolumn{13}{|c|}{ MINORS for comparative studies } \\
\hline & & 1 & 2 & 3 & 4 & 5 & 6 & 7 & 8 & 9 & 10 & 11 & 12 & Score \\
\hline 1 & Cui et al., 2020 [13] & 2 & 1 & 0 & 1 & 0 & 1 & 2 & 0 & 0 & 0 & 0 & 2 & 9 \\
\hline 2 & Meehan et al., 2020 [14] & 2 & 0 & 0 & 1 & 0 & 2 & 2 & 0 & 0 & 1 & 1 & 2 & 11 \\
\hline 3 & Wilms et al., 2020 [16] & 2 & 0 & 2 & 1 & 0 & 2 & 2 & 0 & 0 & 1 & 2 & 2 & 14 \\
\hline 4 & Zhao et al., 2020 [17] & 1 & 2 & 2 & 0 & 0 & 1 & 2 & 0 & 1 & 1 & 2 & 2 & 14 \\
\hline 5 & Chen et al., 2019 [19] & 2 & 1 & 2 & 2 & 0 & 1 & 2 & 0 & 2 & 0 & 1 & 2 & 15 \\
\hline 6 & Kouketsu et al., 2019 [21] & 2 & 1 & 2 & 0 & 0 & 0 & 2 & 0 & 2 & 1 & 1 & 2 & 13 \\
\hline 7 & Weber et al., 2019 [48] & 2 & 1 & 2 & 2 & 2 & 2 & 2 & 0 & 2 & 0 & 1 & 2 & 18 \\
\hline 8 & $\begin{array}{l}\text { Stasikowska-Kanicka et al., } \\
2018 \text { [26] }\end{array}$ & 2 & 2 & 2 & 0 & 0 & 1 & 2 & 0 & 1 & 1 & 1 & 2 & 14 \\
\hline 9 & $\begin{array}{l}\text { Stasikowska-Kanicka et al., } \\
2018 \text { [27] }\end{array}$ & 2 & 2 & 2 & 0 & 0 & 1 & 2 & 0 & 1 & 1 & 1 & 2 & 14 \\
\hline 10 & Weber et al., 2017 [40] & 2 & 1 & 2 & 0 & 0 & 1 & 2 & 0 & 1 & 1 & 1 & 2 & 13 \\
\hline 11 & Malaspina et al., 2011 [47] & 1 & 2 & 2 & 0 & 0 & 1 & 2 & 0 & 1 & 1 & 1 & 2 & 13 \\
\hline
\end{tabular}

1 - a clearly stated aim, 2 -inclusion of consecutive patients, 3-prospective data collection, 4 - endpoints appropriate to the aim of the study, 5 - unbiased assessment of the study endpoint, 6 - follow-up period appropriate to the aim of the study, 7 - loss to follow up less than $5 \%$, 8 - prospective calculation of the study size, 9 - an adequate control group, 10 - contemporary groups, 11 - baseline equivalence of groups, 12 - adequate statistical analyses; score: 0 - not reported, 1 -reported but inadequate, 2 -reported and adequate; the ideal global score for comparative studies is 24.

tration [39], recurrence [42], disease-specific survival [45], disease-free survival $[16,20,45]$, recurrence-free survival [42], overall survival [16, 24, 31, 35, 42], progression-free survival [24] and poor survival [26]. Lin et al. suggested the possibility of using PD-L1 as a prognostic factor especially in smokers and men [44]. No correlation was found between the PD-L1 protein expression and betel chewing, alcohol consumption, perineural invasion, depth of invasion, treatment, or distant metastases [41, 44]. A high expression is associated with better overall survival [30]. In addition, the presence of PD-L1 and TILs expression has been correlated with better outcome in patients with locally advanced OSCC. In those cases, the risk of recurrence was lower and survival was improved [24, 35].

B7-DC/PD-L2 protein (programmed cell death 1 ligand 2/cluster of differentiation 273/CD273/PDCD1LG2/ B7DC/Btdc/PDCD1L2/PDL2/bA574F11.2) is encoded by the PDCD1LG2 gene on chromosome 9 (locus 9p24.1). The B7DC protein has genes parallel to B7-1 ligand in 23\%. PD-L2 has three domains: immunoglobulin constant-like domain (IgC; extracellular), the variable-like domain (IgV; extracellular) and homology domain for PD-1 [8, 57]. Intracellular structures are poorly studied [50-52]. PD-L2 protein is found on dendritic cells, B lymphocytes, Th2 cells, monocytes, macrophages, mast cells, hepatocytes and endothelial cells. This protein suppresses the immune system by inhibiting the T cell response through PD-1 binding. The presence of this molecule on tumour cells may cause the tumour resistance to the immune system $[7,8,58]$. PD-L2 expression was correlated with stage, but not associated with tumour size, nodal metastases, grade, progressionfree survival or overall survival in OSCC [33, 35, 48].
Programmed cell death protein 1 receptor is a transmembrane protein (PD-1/cluster of differentiation 279/ CD279; 50-55 kDa) encoded by the PDCD1 gene on chromosome 2 (locus 2q37.3). It consists of five domains including ITIM (immunoreceptor tyrosine-based inhibitory motif) and ITSM (immunoreceptor tyrosine-based switch motif). The IgV domain has genes parallel to CTLA-4 receptor in $21-33 \%$ and to CD28 receptor in $15.6 \%$ [8, 59]. The PD-1 receptor is found in activated T cells, B lymphocytes, NK cells, mast cells, macrophages and dendritic cells [60]. PD-1 proteins were not detected in immature T lymphocytes. The presence of PD-1 protein on TILs was revealed more frequently in comparison with peripheral blood. This was regardless of the patient's age or HPV infection [61]. PD-1 generates an inhibitory signal that regulates the functions of $\mathrm{T}$ lymphocytes. This receptor has two known ligands: PD-L1 and PD-L2 [58]. Programmed cell death protein 1 receptor does not directly affect apoptosis or cell survival. PD-1 signals regulate the cellular response, but this is not completely clear. The signalling process is different in $\mathrm{B}$ and $\mathrm{T}$ lymphocytes. After binding by the PD-1 receptor, the ligand is followed by phosphorylation of tyrosine in the ITSM domain and recruitment of the SHP-2 (Src homology region 2 domain-containing phosphatase-1) and SHP-1 signal molecules (Src homology region 2 domain-containing phosphatase-2). This blocks the activation of PI-3K molecules (phosphatidylinositol 3-kinase) and ZAP70 (zeta chain-associated protein kinase 70) [58]. Activation of SHP-2 causes dephosphorylation of the molecule involved in TCR receptor activity and as a result reduces signal and cytokine synthesis [58]. Higher prevalence of PD-1 receptors on 
activated lymphocytes suggests that it is more important than the CTLA-4 receptor pathway [62]. An inflammatory reaction induces an increase in the expression of PD-L1 and PD-L2 proteins. They can serve as a feedback mechanism to reduce $T$ cell responses in tissues and protect them from auto-aggressive damage. The binding of PD-L1 ligand to PD-1 receptor leads to the inhibition of proliferation and the reduction of IFN- $\gamma$ and IL-10 cytokine secretion (interleukin 10) by about $80 \%$, and IL-2 (interleukin 2) to the threshold below the reference values. This inhibits the proliferation of lymphocytes and promotes the survival of the cancer cell [63]. The level of PD-1 protein is high on activated lymphocytes, but it quickly decreases after removal of the antigen. However, when lymphocytes have to contend with chronic inflammation (infection or cancer), the expression of PD-1 protein is still high, which causes "exhaustion" of lymphocytes. A high oestrogen level can also induce PD-1 receptors on T cells and APC [64]. The inhibitory effect was found on Th (CD4+; helper cells) and Tc (CD8+; cytotoxic cells) lymphocytes [65]. The presence of the PD-1 protein expression has been demonstrated in bladder cancer [65] and pregnancy-associated melanoma [66]. In OSCC, PD-1 protein expression was correlated with age [30], stage [30], nodal metastases [30], perineural invasion [30] and not related to the disease-free survival [30], recurrencefree survival [42] or overall survival [30, 42]. PD-1 signalling was strongly enriched in never-smokers and neverdrinkers [30]. The role of the PD-1/PD-L1 pathway is not limited to the pathogenesis of tumours. This pathway is important in various diseases: insulin-dependent diabetes mellitus, lupus erythematosus, myocarditis, inflammation of the brain and spinal cord, rheumatoid arthritis and inflammatory bowel diseases [67].

\section{B7 pathway inhibition in cancers and OSCC}

The B7/CD28 pathway influences the regulation of the immune response by limiting the time and strength of the inflammatory response. Although the co-stimulation mechanism of the B7-CD28 pathway is not known, monoclonal antibodies are currently used in targeted therapies of malignant tumours, autoimmune and infectious diseases. In cancer, CTLA-4 and PD-1 receptors are blocked by ligands (B7-1 and B7-2 for CTLA-4, and PD-L1 and PD-L2 for PD-1). As a result of ligand-receptor binding, neoplastic lesions are not recognized by the immune system. The function of monoclonal antibodies is to block CTLA-4 and PD-1 receptors. As a result, T lymphocytes re-recognize tumour antigens. The immunomodulatory drugs that block the CTLA-4 protein are ipilimumab and tremelimumab, while the PD-1 is blocked by nivolumab, pembrolizumab, pidilizumab, BMS-936559, MEDI4736 (durvalumab) and MPDL3280A [49, 56, 68-72]. They are mainly used in the treatment of melanoma $[66,70]$, lung cancer $[66,70]$, genitourinary cancer [66] and prostate cancer [72]. Targeted immunotherapy in head and neck melanoma improved survival. Ipilimumab and nivolumab had a better effect together than in monotherapy [56]. Nivolumab was used in a clinical trial of 296 melanoma, non-small-cell lung cancer, prostate cancer, renal cancer, and colorectal cancer patients. The positive tumour response (expression of PD-L1 in > 5\% of cells) was seen in $18 \%$ of patients with non-small cell lung cancer, $28 \%$ with melanoma and in $27 \%$ with renal-cell carcinoma [56]. In Carbognin et al. study, nivolumab, pembrolizumab and MPDL3280A were studied in patients with melanoma, lung and genitourinary cancers. The overall response rate was significantly higher in patients with positive PD-L1 expression [68].

The role of immunomodulatory drugs in the treatment of oral cancer remains unclear and requires more research. The oral squamous cell carcinoma is highly immunosuppressive. An anti-PD-1 monoclonal antibody therapy may result in better clinical efficacy in OSCC patients [73-75]. Foy et al. studied the clinical response to pembrolizumab in HPV-negative oral squamous cell carcinoma. The PD-L1 protein was overexpressed and the score of response to pembrolizumab was higher in neversmokers and never-drinkers than in smokers and drinkers, although the mutational load was lower in neversmokers and drinkers. The main difference between oral squamous cell carcinoma in never-smokers and neverdrinkers when compared to smokers and drinkers, lies in the immune microenvironment, suggesting a higher clinical benefit of PD-L1 inhibition in oral cancer in neversmokers and drinkers. The immune checkpoint inhibitors can probably extend the survival of many patients [32].

\section{Conclusions}

The biology of squamous cell carcinoma is unknown. The search for new molecular markers is extremely important. Components of the B7 family are potential objects of research. Any mutations in gene encoding PD-L1 and quantitative changes in the status of PD-L1 protein may have an impact on the prognosis of oral squamous cell carcinoma.

\section{Conflict of interest}

The authors declare no conflict of interest.

\section{References}

1. Ferlay J, Soerjomataram I, Dikshit R, et al. Cancer incidence and mortality worldwide: sources, methods and major patterns in GLOBOCAN 2012. Int J Cancer 2015; 136: E359-86.

2. Global Burden of Disease Cancer Collaboration. Global, regional, and national cancer incidence, mortality, years of life lost, years lived with disability, and disability-adjusted life-years for 29 Cancer Groups, 1990 to 2017: a systematic analysis for the global burden of disease study. JAMA Oncol 2019; 5: 1749-68. 
3. Johnson NW, Jayasekara P, Amarasinghe AA, et al. Squamous cell carcinoma and precursor lesions of the oral cavity: epidemiology and aetiology. Periodontology 2000 2011; 57: 19-37.

4. Starzyńska A, Sejda A, Adamska P, et al. Prognostic value of the PIK3CA, AKT, PTEN mutations in oral squamous cell carcinoma: literature review. Arch Med Sci doi:10.5114/ aoms.2018.77859.

5. Zou W. Immunosuppressive networks in the tumour environment and their therapeutic relevance. Nat Rev Cancer 2005; 5: 263-74.

6. Song MK, Park BB, Uhm J. Understanding immune evasion and therapeutic targeting associated with PD-1/PD-L1 pathway in diffuse large B-cell lymphoma. Int J Mol Sci 2019; 20: 1326.

7. Ni L, Dong D. New B7 family checkpoints in human cancers. Mol Cancer Ther 2017; 16: 1203-11.

8. Schildberg FA, Klein SR, Freeman GJ, et al. Coinhibitory pathways in the B7-CD28 ligand-receptor family. Immunity 2016; 44: $955-72$.

9. Xiao Y, Freeman GJ. A new B7:CD28 family checkpoint target for cancer immunotherapy: HHLA2. Clin Cancer Res 2015; 21: 2201-3.

10. Liberati A, Altman DG, Tetzlaff J, et al. The PRISMA statement for reporting systematic reviews and meta-analyses of studies that evaluate health care interventions: explanation and elaboration. Ann Intern Med 2009; 151: 65-94.

11. Higgins JPT, Altman DG, Gøtzsche PC, et al. The Cochrane Collaboration's tool for assessing risk of bias in randomised trials. Br Med J 2011; 343: d5928.

12. Slim K, Nini E, Forestier D, et al. Methodological index for non-randomized studies (minors): development and validation of a new instrument. ANZ J Surg 2003; 73: 712-6.

13. Cui $B$, Chen J, Luo $M$, et al. Protein kinase $D 3$ regulates the expression of the immunosuppressive protein, PDL1, through STAT1/STAT3 signaling. Int J Oncol 2020; 56: 909-20.

14. Meehan K, Leslie C, Lucas M, et al. Characterization of the immune profile of oral tongue squamous cell carcinomas with advancing disease. Cancer Med 2020; 9: 4791-807.

15. Quan H, Shan Z, Liu Z, et al. The repertoire of tumor-infiltrating lymphocytes within the microenvironment of oral squamous cell carcinoma reveals immune dysfunction. Cancer Immunol Immunother 2020; 69: 465-76.

16. Wilms T, Gu X, Boldrup L, et al. PD-L1 in squamous cell carcinoma of the oral tongue shows gender-specific association with prognosis. Oral Dis 2020; doi: 10.1111/odi.13414.

17. Zhao L, Li P, Zhao L, et al. Expression and clinical value of PD-L1 which is regulated by BRD4 in tongue squamous cell carcinoma. J Cell Biochem 2020; 121: 1855-69.

18. Ahmadi N, Gao K, Chia N, et al. Association of PD-L1 expression in oral squamous cell carcinoma with smoking, sex, and p53 expression. Oral Surg Oral Med Oral Pathol Oral Radiol 2019; 128: 631-8.

19. Chen XJ, Tan YQ, Zhang N, et al. Expression of programmed cell death-ligand 1 in oral squamous cell carcinoma and oral leukoplakia is associated with disease progress and CD8+ tumor-infiltrating lymphocytes. Pathol Res Pract 2019; 215: 152418.

20. de Vincente JC, Rodríguez-Santamarta T, Rodrigo JP, et al. PD-L1 expression in tumor cells is an independent unfavorable prognostic factor in oral squamous cell carcinoma. Cancer Epidemiol Biomarkers Prev 2019; 28: 546-54.

21. Kouketsu A, Sato I, Oikawa M, et al. Expression of immunoregulatory molecules PD-L1 and PD-1 in oral cancer and precancerous lesions: a cohort study of Japanese patients. J Craniomaxillofac Surg 2019; 47: 33-40.

22. Takahashi H, Sakakura K, Arisaka Y, et al. Clinical and biological significance of PD-L1 expression within the tumor microenvironment of oral squamous cell carcinoma. Anticancer Res 2019; 39: 3039-46.

23. Tojyo I, Shintani Y, Nakanishi T, et al. PD-L1 expression correlated with p53 expression in oral squamous cell carcinoma. Maxillofac Plast Reconstr Surg 2019; 41: 56.

24. Hanna GJ, Woo SB, Li YY, et al. PD-L1 expression is associated with improved survival and lower recurrence risk in young women with oral cavity squamous cell carcinoma. Int J Oral Maxillofac Surg 2018; 47: 568-77.

25. Maruse Y, Kawano S, Jinno T, et al. Significant association of increased PD-L1 and PD-1 expression with nodal metastasis and a poor prognosis in oral squamot alus cell carcinoma. Int J Oral Maxillofac Surg 2018; 47: 836-45.

26. Stasikowska-Kanicka O, Wagrowska-Danilewicz M, Danilewicz M. Immunohistochemical analysis of Foxp3+, CD4+, CD8+ cell infiltrates and PD-L1 in oral squamous cell carcinoma. Pathol Oncol Res 2018; 24: 497-505.

27. Stasikowska-Kanicka O, Wagrowska-Danilewicz M, Danilewicz M. CD8+ and CD163+ infiltrating cells and PD-L1 immunoexpression in oral leukoplakia and oral carcinoma. APMIS 2018; 126: 732-8.

28. Udeabor SE, Adisa AO, Orlowska A, et al. Assessment of programmed cell death proteins in oral squamous cell carcinoma. Afr J Biomed Res 2018; 21: 11-3.

29. Wirsing AM, Ervik IK, Seppola M, et al. Presence of high-endothelial venules correlates with a favorable immune microenvironment in oral squamous cell carcinoma. Mod Pathol 2018; 31: 910-22.

30. Ahn H, Yang JM, Kim H, et al. Clinicopathologic implications of the miR-197/PD-L1 axis in oral squamous cell carcinoma. Oncotarget 2017; 8: 66178-94.

31. Feng Z, Bethmann D, Kappler M, et al. Multiparametric immune profiling in HPV-oral squamous cell cancer. JCI Insight 2017; 2: 93652

32. Foy JP, Bertolus C, Michallet MC, et al. The immune microenvironment of HPV-negative oral squamous cell carcinoma from never-smokers and never-drinkers patients suggests higher clinical benefit of IDO1 and PD1/PD-L1 blockade. Ann Oncol 2017; 28: 1934-41.

33. Groeger S, Domann E, Gonzales JR, et al. B7-H1 and B7-DC receptors of oral squamous carcinoma cells are upregulated by Porphyromonas gingivalis. Immunobiology 2011; 216: 1302-10.

34. Hirai M, Kitahara H, Kobayashi Y, et al. Regulation of PD-L1 expression in a high-grade invasive human oral squamous cell carcinoma microenvironment. Int J Oncol 2017; 50: 41-8.

35. Kogashiwa Y, Yasuda M, Sakurai H, et al. PD-L1 expression confers better prognosis in locally advanced oral squamous cell carcinoma. Anticancer Res 2017; 37: 1417-24.

36. Kubota K, Moriyama M, Furukawa S, et al. CD163+CD204+ tumor-associated macrophages contribute to T cell regulation via interleukin-10 and PD-L1 production in oral squamous cell carcinoma. Sci Rep 2017; 7: 1755.

37. Mattox AK, Lee J, Westra WH, et al. PD-1 expression in head and neck squamous cell carcinomas derives primarily from functionally anergic CD4+ TILs in the presence of PD -L1+ TAMs. Cancer Res 2017; 77: 6365-74.

38. Takakura H, Domae S, Ono T, et al. The Immunological impact of chemotherapy on the tumor microenvironment of 
oral squamous cell carcinoma. Acta Med Okayama 2017; 71: 219-26.

39. Troeltzsch M, Woodlock T, Pianka A. Is there evidence for the presence and relevance of the PD-1/PD-L1 pathway in oral squamous cell carcinoma? Hints from an immunohistochemical study. J Oral Maxillofac Surg 2017; 75: 969-77.

40. Weber M, Wehrhan F, Baran C, et al. PD-L1 expression in tumor tissue and peripheral blood of patients with oral squamous cell carcinoma. Oncotarget 2017; 8: 112584-97.

41. Satgunaseelan L, Gupta R, Madore J, et al. Programmed cell death -ligand 1 expression in oral squamous cell carcinoma is associated with an inflammatory phenotype. Pathology 2016; 48: 574-80.

42. Straub M, Drecoll E, Pfarr N, et al. CD274/PD-L1 gene amplification and PD-L1 protein expression are common events in squamous cell carcinoma of the oral cavity. Oncotarget 2016; 7: 12024-34.

43. Chen TC, Wu CT, Wang CP, et al. Associations among pretreatment tumor necrosis and the expression of HIF-1alpha and PD-L1 in advanced oral squamous cell carcinoma and the prognostic impact thereof. Oral Oncol 2015; 51: 1004-10.

44. Lin YM, Sung WW, Hsieh MJ, et al. High PD-L1 expression correlates with metastasis and poor prognosis in oral squamous cell carcinoma. PLoS One 2015; 10: e0142656.

45. Oliveira-Costa JP, de Carvalho AF, da Silveira GG, et al. Gene expression patterns through oral squamous cell carcinoma development: PD-L1 expression in primary tumor and circulating tumor cells. Oncotarget 2015; 6: 20902-20.

46. Cho YA, Yoon HJ, Lee Jl, et al. Relationship between the expressions of PD-L1 and tumor-infiltrating lymphocytes in oral squamous cell carcinoma. Oral Oncol 2011; 47: 1148-53.

47. Malaspina TS, Gasparoto TH, Costa MR, et al. Enhanced programmed death 1 (PD-1) and PD-1 ligand (PD-L1) expression in patients with actinic cheilitis and oral squamous cell carcinoma. Cancer Immunol Immunother 2011; 60: 965-74.

48. Weber M, Wehrhan F, Baran C, et al. Prognostic significance of PD-L2 expression in patients with oral squamous cell carcinoma-a comparison to the PD-L1 expression profile. Cancer Med 2019; 8: 1124-34

49. Mashima E, Inoue A, Sakuragi Y, et al. Nivolumab in the treatment of malignant melanoma: review of the literature. Therapy 2015; 8: 2045-52.

50. Ohigashi Y, Sho M, Yamada Y, et al. Clinical significance of programmed death-1 ligand-1 and programmed death-1 ligand-2 expression in human esophageal cancer. Clin Cancer Res 2005; 11: 2947-53.

51. Nomi T, Sho M, Akahori T, et al. Clinical significance and therapeutic potential of the programmed death-1 ligand/ programmed death-1 pathway in human pancreatic cancer. Clin Cancer Res 2007; 13: 2151-7.

52. Hamanishi J, Mandai M, Iwasaki M, et al. Programmed cell death 1 ligand 1 and tumor-infiltrating CD8+ T lymphocytes are prognostic factors of human ovarian cancer. Proc Nat Acad Sci USA 2007; 104: 3360-5.

53. Wang L, Ma Q, Chen X, et al. Clinical significance of B7-H1 and B7-1 expressions in pancreatic carcinoma. World J Surg 2010; 34: 1059-65.

54. Zgodzinski W, Grywalska E, Zinkiewicz K, et al. Peripheral blood T lymphocytes are downregulated by the PD-1/PD-L1 axis in advanced gastric cancer. Arch Med Sci 2019; 15: 774 83.

55. Germanidis G, Argentou N, Hytiroglou P, et al. Liver FOXP3 and PD1/PDL1 expression is down-regulated in chronic HBV hepatitis on maintained remission related to the degree of inflammation. Front Immunol 2013; 4: 207.
56. Swanson MS, Sinha UK. Rationale for combined blockade of PD-1 and CTLA-4 in advanced head and neck squamous cell cancer - review of current data. Oral Oncol 2015; 51: 12-5.

57. Moreira G, Fulgęncio LB, De Mendonca EF, et al. T regulatory cell markers in oral squamous cell carcinoma: relationship with survival and tumor aggressiveness. Oncol Lett 2010; 1: $127-32$.

58. Okazaki T, Wang J. PD-1/PD-L pathway and autoimmunity. Autoimmunity 2005; 38: 353-7.

59. Okazaki T, Honjo T. PD-1 and PD-1 ligands: from discovery to clinical application. Int Immunol 2007; 19: 813-24.

60. Sharpe AH, Freeman GJ. The B7-CD28 superfamily. Nat Rev Immunol 2002; 2: 116-26.

61. Montler R, Bell RB, Thalhofer C, et al. OX40, PD-1 and CTLA-4 are selectively expressed on tumor-infiltrating $T$ cells in head and neck cancer. Clin Transl Immunol 2016; 5: e70.

62. Freeman GJ, Long AJ, Iwai Y, et al. Engagement of the PD-1 immunoinhibitory receptor by a novel B7 family member leads to negative regulation of lymphocyte activation. J Exp Med 2000; 192: 1027-34.

63. Gianchecchi E, Delfino DV, Fierabracci A. Recent insights into the role of the PD-1/PD-L1 pathway in immunological tolerance and autoimmunity. Autoimmun Rev 2013; 12: 1091-100.

64. Polanczyk MJ, Hopke C, Vandenbark AA, et al. Treg suppressive activity involves estrogen-dependent expression of programmed death-1 (PD-1). Int Immunol 2007; 19: 337-43.

65. Inman BA, Longo TA, Ramalingam S, et al. Atezolizumab: a PD-L1-blocking antibody for bladder cancer. Clin Cancer Res 2017; 23: 1886-90.

66. Ko JS, Gastman BR, Conic R, et al. Decreased T-Cell programmed death receptor-1 expression in pregnancy-associated melanoma. Am J Dermatopathol 2019; 41: 180-7.

67. Guery T, Roumier C, Berthon C, et al. B7-H3 protein expression in acute myeloid leukemia. Cancer Med 2015; 4: 1879-83.

68. Carbognin L, Pilotto S, Milella M, et al. Differential activity of nivolumab, pembrolizumab and MPDL3280A according to the tumor expression of programmed death-ligand-1 (PD-L1): sensitivity analysis of trials in melanoma, lung and genitourinary cancers. PLoS One 2015; 10: e0130142.

69. Berghoff AS, Ricken G, Widhalm G, et al. PD1 (CD279) and PD-L1 (CD274, B7H1) expression in primary central nervous system lymphomas (PCNSL). Clin Neuropathol 2014; 33: 42-9.

70. Mahoney KM, Freeman GJ, McDermott DF. The next immunecheckpoint inhibitors: PD-1/PD-L1 blockade in melanoma. Clin Ther 2015; 37: 764-82.

71. Planchard D, Yokoi T, McCleod MJ, et al. A phase III study of durvalumab (MEDI4736) with or without tremelimumab for previously treated patients with advanced NSCLC: rationale and protocol design of the ARCTIC study. Clin Lung Cancer 2016; 17: 232-6

72. Gao J, Ward JF, Pettaway CA, et al. VISTA is an inhibitory immune checkpoint that is increased after ipilimumab therapy in patients with prostate cancer. Nat Med 2017; 23: 551-5.

73. Ibrahim R, Stewart R, Shalabi A. PD-L1 blockade for cancer treatment: MEDI4736. Semin Oncol 2015; 42: 474-83.

74. Lyford-Pike S, Peng S, Young GD, et al. Evidence for a role of the PD-1:PD-L1 pathway in immune resistance of HPVassociated head and neck squamous cell carcinoma. Cancer Res 2013; 73: 1733-41.

75. Zandberg DP, Strome SE. The role of the PD-L1:PD-1 pathway in squamous cell carcinoma of the head and neck. Oral Oncol 2017; 50: 627-32. 